\title{
Post Kala-azar Dermal Leishmaniasis Presenting with Visceral Leishmaniasis
}

\section{Maharshi Trivedi, Anju Aggarwal*, Parul Gupta and Sonal Sharma}

Department of Pediatrics and Pathology, University College of Medical Sciences, Guru Tegh Bahadur Hospital, New Delhi, India

\begin{abstract}
Post kala-azar dermal leishmaniasis is a dermal complication occurring months after treatment of visceral leishmaniasis. A 10 year old boy, resident of kalaazar endemic area presented with multiple hypopigmented macules over both legs and abdomen for 3 years. Child had splenomegaly. Splenic puncture revealed LD bodies and RK 39 antibody positivity. Skin revealed unremarkable epidermis and dermal perivascular inflammation in $100 \mathrm{X}$ magnification. Leishmania donovani body was seen 1000X magnification. Presentation of PKDL with VL is rare. Child was treated with liposomal amphotericin B. Skin lesions decreased and spleen size regressed after treatment.
\end{abstract}

Keywords: Post kala azar dermal leishmaniasis; Visceral leishmaniasis; Child

\section{Introduction}

Leishmaniasis is a group of diseases caused by various species of the protozoan parasites of the genus Leishmania. Leishmaniasis can be categorized in 3 major forms ranging in severity from spontaneously healing skin ulcers in cutaneous leishmaniasis (CL), destructive mucocutaneousleishmaniasis (MCL) to fatal visceral leishmaniasis (VL). Post-kala-azar dermal leishmanisis (PKDL) is a dermatologic manifestation which usually occurs months to years after resolution of visceral leishmaniasis (VL) caused by Leishmania donovani. Upto $10-15 \%$ cases of PKDL do not have history of previous visceral leishmaniasis, may be they have had a subclinical infection $[1,2]$. Presentation of PKDL with VL is rare, there are hardly any case reports in which presentation of post kalaazar visceral leishmaniasis is seen with visceral leishmaniasis, hence a gap in knowledge about this presentation.

\section{Case Presentation}

A 10 year old boy resident of Bihar presented with complaints of non-itchy hypopigmented macules over bilateral legs present for last 3 years. There was no history of weight loss and no history of tuberculosis and leprosy contact. On general physical examinations. Multiple hypo pigmented macular lesions were present over both legs and over trunk (Figure 1). They were not associated with numbness; tingling, burning sensations. On abdomen examination spleen $(5.5 \mathrm{cms})$ was palpable.

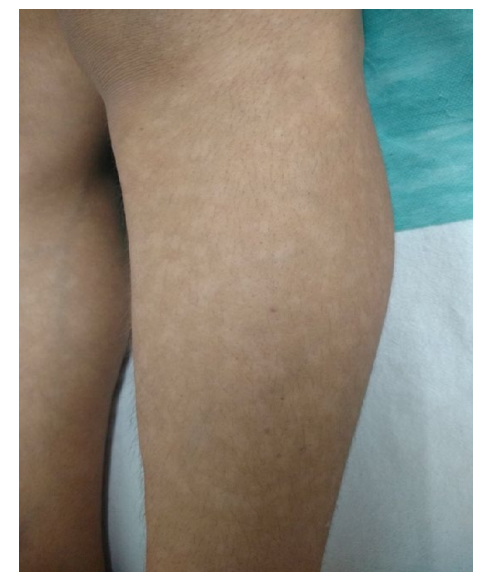

Figure 1: Hypopigmneted lesions over the legs of the child.

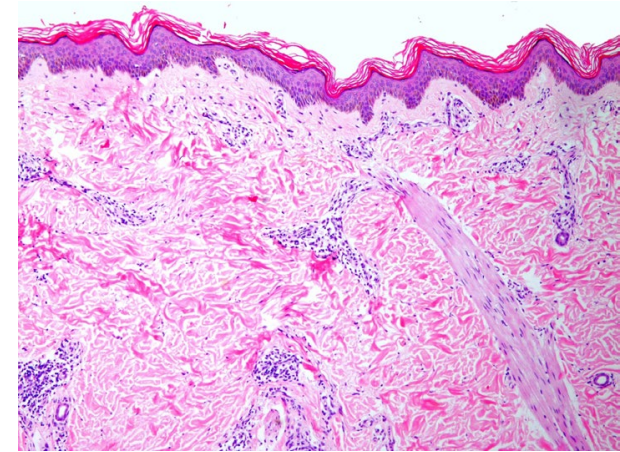

Figure 2: Section from skin with unremarkable epidermis and dermal perivascular inflammation, $\mathrm{H} \& \mathrm{E} \times 100$.

There was no hepatomegaly or free fluid in the abdomen. Rest of the systemic examination did not reveal any abnormality. On laboratory evaluations hemoglobin was $9.1 \mathrm{gm} / \mathrm{dl}$, TLC was $3600 / \mathrm{mm}^{3}$, platelet counts were $1,78,000 / \mathrm{mm}^{3}$ and peripheral smear did not reveal any abnormality. Renal function tests were normal. Serology for HIV infection was negative. Splenic puncture revealed LD bodies and RK 39 antibody positivity. Skin biopsy from macule was suggestive of post kala-azar dermal leishmaniasis. Skin revealed unremarkable epidermis and dermal perivascular inflammation in $100 \mathrm{X}$ magnification. Leishmaniadonovani body was seen on 1000X magnification (Figure 2). As per NVBDCP National vector borne diseases control program [3] child was treated liposomal amphotericin B @5 mg/ $\mathrm{kg} /$ dose twice weekly for 3 weeks. Complete blood counts were done on weekly follow up. After treatment with liposomal amphotericin B child became afebrile, hypopigmented macules were becoming less apparent and decreased in number. Spleen size reduced to $1.5 \mathrm{~cm}$ at the end of $3 \mathrm{rd}$ week along with improvement in appetite and activity of the child (Figure 3).

*Corresponding author: Anju Aggarwal, Department of Pediatrics and Pathology University College of Medical Sciences, Guru Tegh Bahadur Hospital, New Delhi-110058, India, Tel: 09910329791; E-mail: aanju67@gmail.com

Received: February 05, 2017; Accepted: February 18, 2017; Published: February 25,2017

Citation: Trivedi M, Aggarwal A, Gupta P, Sharma S (2017) Post Kala-azar Dermal Leishmaniasis Presenting with Visceral Leishmaniasis. J Infect Dis Med 2: 105. doi: 10.4172/2576-1420.1000105

Copyright: $\odot 2017$ Trivedi M, et al. This is an open-access article distributed under the terms of the Creative Commons Attribution License, which permits unrestricted use, distribution, and reproduction in any medium, provided the original author and source are credited. 


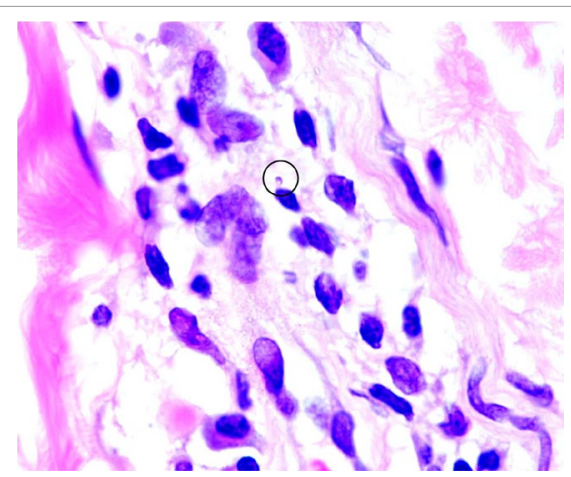

Figure 3: LD body (encircled) inside a histiocyte in perivascular infiltrate, H\&E x1000.

\section{Discussion and Conclusion}

Post kala-azar dermal leishmaniasis (PKDL) is a dermal complication resulting after treatment of VL. Clinically the lesions of PKDL may vary from hypo-pigmented macules to infiltrated papule and nodules, starting from around mouth and spreading to other parts of body depending upon severity. Three major representation of skin manifestations are described i) erythematous indurated lesions on butterfly area of face ii) multiple symmetrical hypo-pigmented macules with irregular margins having generalized distribution to extremities and trunk iii) combination of papules, nodules and plaques. In $15 \%$ of instances, mucosal involvement has been observed [1].

The presentation of PKDL differs in clinical forms and as well as duration after VL that occurs. In India $5-15 \%$ of VL cases are complicated by PKDL occurring months to several years (2-3 years) after remission of infection, while in Sudan $50-60 \%$ of cured VL cases are followed by PKDL within weeks or months (0-6 months) [4]. Post kala-azar dermal leishmaniasis has been reported in patients of visceral leishmaniasis treated with sodium stibogluconate, paromomycin, miltefosin, amphotericin B [5-7]. Cases of PKDL with rare presentation as macroglosia and along with oral mucosal involvement have been reported $[7,8]$. Up to $15-20 \%$ of PKDL cases had no preceding history of visceral leishmaniasis, suggestive of subclinical infection. However, these cases did not have any evidence of visceral involvement at the time of presentation [4]. In our case the child had simultaneous manifestations of visceral leishmaniasis and post kala-azar dermal leishmaniasis.
Demonstration of parasites inslit skin smear or by culture of the dermal tissue is considered to be the gold standard but such methods are invasive and less sensitive $(58 \%)$ because of low parasite burden and, thus, not feasible in field conditions [9]. On the contrary, recent availability of rapid sero diagnostic tools, like the rK39, has revolutionized the diagnosis of VL as well as PKDL (sensitivity of around 95\% for polymorphic PKDL and 78\% for macular variety) [9]. As a result, this simple and rapid test has been recommended and is being widely used in detection of both VL and PKDL in the elimination programs of India, Bangladesh, and Nepal.

The complications can result when these lesions are not treated or mucous membranes are affected. Treatment is always needed in Indian PKDL; in Sudan most cases will self-cure but severe and chronic cases are treated. Treatment of PKDL is important for control of leishmaniasis. This case highlights dermal manifestations of PKDL presenting with visceral leishmaniasis a rare presentation.

\section{References}

1. Ramesh V, Kaushal H, Mishra AK, Singh R, Salotra P, et al. (2015) Clinicoepidemiological analysis of Post kala-azar dermal leishmaniasis (PKDL) cases in India over last two decades: A hospital based retrospective study. BMC Public Health 15: 1092

2. Ramesh V, Mukherjee A (1995) Post-kala-azar dermal leishmaniasis. Int J Dermatol 34: 85-91.

3. http://nvbdcp.gov.in/kal13.html

4. Zijlstra EE, el-Hassan AM (2001) Leishmaniasis in Sudan. Post kala-azar dermal leishmaniasis. Trans R Soc Trop Med Hyg 95: S59-76.

5. Burza S, Sinha PK, Mahajan R, Sanz MG, Lima MA, et al. (2014) Pos Kala-azar dermal leishmaniasis following treatment with $20 \mathrm{mg} / \mathrm{kg}$ liposomal amphotericin B (Ambisome) for primary visceral leishmaniasis in Bihar, India. PLoS Negl Trop Dis 8: e2611.

6. Pandey K, Das VNR, Singh D, Das S, Lal CS, et al. (2012) Post-kala-azar dermal leishmaniasis in a patient treated with injectable paromomycin for visceral leishmaniasis in India. J Clin Microbiol 50: 1478-1479.

7. Salam MA, Siddiqui MA, Nabi SG, Bhaskar KRH, Mondal D (2013) Postkala-azar dermal leishmaniasis with mucosal involvement: An unusual case presentation including successful treatment with miltefosine. J Health Popul Nutr 31: 294-297.

8. Nikzad A, Nobari H, Firooz A, Dowlati Y (2005) Macroglossia as a manifestation of post-kala-azar dermal leishmaniasis: A case report. Int J Dermatol 44: 129-131.

9. Salotra P, Sreenivas G, Ramesh V, Sundar S (2001) A simple and sensitive test for field diagnosis of post kala-azar dermal leishmaniasis. $\mathrm{Br} \mathrm{J}$ Dermatol 145: 630-632. 